\title{
Directed Fixed Energy Sandpile Model
}

\author{
R. Karmakar and S. S. Manna \\ Satyendra Nath Bose National Centre for Basic Sciences Block-JD, Sector-III, Salt Lake, Kolkata-700098, India
}

\begin{abstract}
We numerically study the directed version of the fixed energy sandpile. On a closed square lattice, the dynamical evolution of a fixed density of sand grains is studied. The activity of the system shows a continuous phase transition around a critical density. While the deterministic version has the set of nontrivial exponents, the stochastic model is characterized by mean field like exponents.
\end{abstract}

PACS numbers: 05.65.+b 05.70.Jk, 45.70.Ht 05.45.Df

Spontaneous emergence of long ranged spatio-temporal correlations under a self-organizing dynamics, in absence of a fine tuning parameter is the basic idea of Selforganized Criticality (SOC). Sandpile models are the prototype models of SOC $1,2,13,4,5,6,6,8,9]$. In sandpile models an integer height variable $h_{i}$ representing the number of grains in the sand column is associated with every site of a regular lattice. The system is driven by adding unit grains of sand at a time. When the height $h_{i}>h_{c}$ the sand column topples and it looses some grains which are distributed among the neighboring sites 1]. This creates an avalanche of sand column topplings and the extent of such activity measures the size of the avalanche. Sand grains go out of the system through a boundary so that in the steady state the fluxes of inflow and outflow currents balance.

On the other hand a fixed energy sandpile (FES) 10, 11, 12, 13] is a sandpile model within a closed system. Therefore the total mass of sand in this system is a conserved quantity. The control parameter is the density $\zeta$ of grains. A stable state has heights varying from 0 to $h_{c}-1$ and is called an inactive state, whereas, any height configuration that has at least one unstable site is said to be in the active state. The dynamics of the system starts with a random distribution of $N=\zeta L^{2}$ grains. Initially some sites may be unstable, which topple. Consequently some of the neighboring sites may topple again and the activity continues. For an infinitely large system there exists a critical threshold $\zeta_{c}$ such that if $\zeta<\zeta_{c}$ the activity terminates and the system gets absorbed in an inactive state where as for $\zeta>\zeta_{c}$ the activity of the system fluctuates but maintains a steady mean value [10]. Therefore $\zeta_{c}$ is the critical point of a continuous phase transition from an absorbed phase to an active phase.

After the dynamics starts, the system takes some time to relax to the steady state. The activity at a certain time is measured by the fraction $\rho$ of lattice sites which are unstable at that time. In general the mean activity $\langle\rho\rangle$ is a function of the density $\zeta$ i.e., the deviation from the critical point $\Delta=\zeta-\zeta_{c}$ and also the system size $L$. The simultaneous dependence of activity on $\Delta$ and $L$ is expressed by the following scaling form:

$$
\langle\rho(\Delta, L)\rangle=L^{-\beta / \nu_{\perp}} \mathcal{G}\left(L^{1 / \nu_{\perp}} \Delta\right)
$$

where $\mathcal{G}(x)$ is an universal scaling function such that $\mathcal{G}(x) \rightarrow x^{\beta}$ when $x>>1$. This implies that for a certain range of $\Delta$ if $L$ is so large that $L^{1 / \nu_{\perp}} \Delta>>1$ then $\langle\rho(\Delta, L)\rangle$ is independent of $L$ and depends solely on $\Delta$ as $\langle\rho(\Delta, L)\rangle \sim \Delta^{\beta}$. Naturally $\beta$ is the order parameter exponent for the transition. On the other hand when $x<<1, \mathcal{G}(x) \rightarrow$ constant, independent of both $\Delta$ and $L$ implies that right at the critical point $\zeta=\zeta_{c}$ the order parameter varies with the system size as: $\langle\rho(L)\rangle \sim L^{-\beta / \nu_{\perp}}$, independent of $\Delta$.

To which universality class the FES model exponents should correspond to? Intensive research has been done to study the universality class of the phase transitions in FES models. It has been suggested that FES belongs to the universality class of the linear interface models (LIM) but not of that of the Directed percolation (DP) universality class 10, 11, 12, 13]. DP is generic for continuous absorbing state transitions in the absence of a conservation law where as in FES there exists a conserved field which is the density and it couples the order parameter, i.e., the mean activity. We, in the present study like to examine if an explicit application of the directional bias to the FES system makes the system behave as DP or it results to another new universality class.

Application of a global directional bias onto a system has been proved to have strong effect on the critical behaviors of various models in Statistical Physics. In directed systems, degrees of freedom of the individual elements is reduced, which shrinks the configuration space of the system compared to undirected system. As a result a directed system is simpler and easily tractable analytically. Examples include Directed percolation [14], Directed Sandpile Model 15, 16, 17], Directed River networks [18 and Directed Self-avoiding walks [19] etc.

We study here the directed fixed energy sandpile (DFES) models on an oriented square lattice placed on the $x-y$ plane, periodic boundary conditions are imposed along both the directions. A preferred direction is imposed onto this system along the $-y$ direction. The critical height of stability of a sand column is fixed at $h_{c}=1$, and on a toppling two grains of sand are distributed to the two neighboring sites along the preferred direction i.e., at the lower-left (LL) and lower-right (LR) positions. 


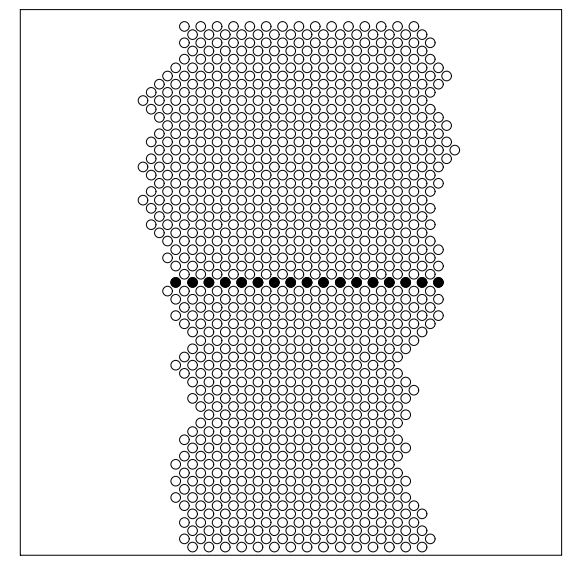

FIG. 1: Filled circles denote the sites on the toppling front in an infinite avalanche of DDFES on an oriented square lattice of size $L=32$. This avalanche is periodic and has a period 64. Horizontal empty circles denote positions of the TF in another 63 time units. The two end sites of the TF fluctuates but maintains a mean distance of $L / 2$.

Two different versions of the model are studied according to the rule by which the two grains are distributed in a toppling: (i) both the LL and LR sites get one grain each, which we call as the deterministic directed fixed energy sandpile model (DDFES), (ii) each of the two grains is distributed randomly to any of the LL or LR positions, and this version is called the stochastic directed fixed energy sandpile (SDFES) model.

We shall first discuss the properties of DDFES. The critical point can be arrived at from an inactive state by adding grains one by one on an initial empty lattice followed by the relaxation of the avalanche. On the average both the size and the life times of the avalanches increase as the density grows. The toppling front (TF) of an avalanche is a set of horizontal contiguous sites which travels downward with unit speed. The length of the TF however fluctuates (Fig. 1). If at an intermediate time the TF has $n$ sites, then at the next time step its length can be only $n-1, n$ or $n+1$. For a finite avalanche the TF first grows from a single site to a certain length and then shrinks to zero. The set of sites covered by the left and right end sites of $\mathrm{TF}$ are the paths of two annihilating random walkers [15]. For a finite avalanche they meet and annihilate, however for an infinite avalanche these two random walkers cannot meet and the best possible way it can be ensured if they can maintain a distance of $L / 2$ on the average from each other.

Therefore the minimum possible sustained activity for DDFES in a system of size $L$ is $\rho=1 /(2 L)$. What is interesting is, on such a system if the density is slightly increased the avalanches created by the additional grains die away and the system maintains the activity of the infinite avalanche. In a sense the system gets locked with this activity for a certain range of grain density. However

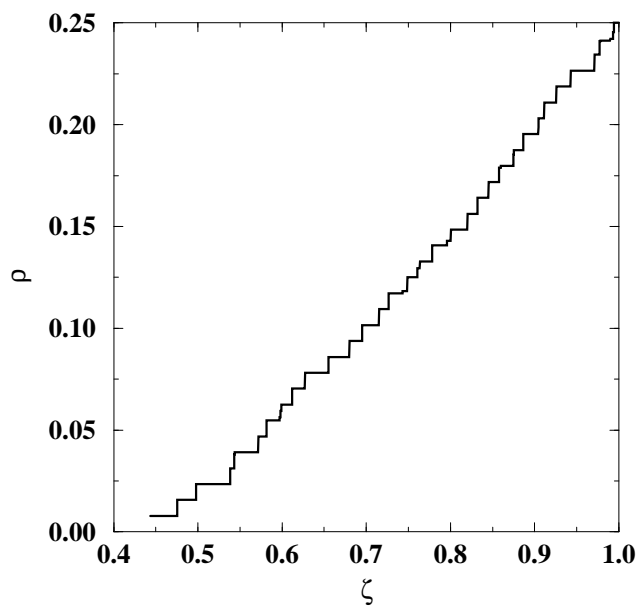

FIG. 2: The mean activity $\langle\rho\rangle$ in a system of size $L=64$ which grows with the density in a step like manner where the step heights are $\Delta \rho=1 /(2 L)$.

on increasing the density even further, a second infinite avalanche is created and both the TFs run simultaneously resulting a sudden jump in the activity by doubling its magnitude to $1 / L$. This continues for some range of grain density which ends at another jump in activity to $3 /(2 L)$. Thus in general the variation of activity is discrete and has a step like variation with step heights $1 /(2 L)$ (Fig. 2 ). As the system size increases the step height decreases to zero and the variation of $\rho$ with $\zeta$ becomes more and more smooth. Similar step-like behavior of the order parameter was also observed in [20].

The configurations at the steady state are periodic and the same detailed distribution of grain numbers at all sites repeat at regular intervals of time. The periodic time is always multiples of $L$ in a $L \times L$ system. For small systems this period has different values for different initial configurations but in most (about 95\%) cases the period is $2 L$ and rarely $L, 3 L, 4 L$ etc. However, for bigger system sizes e.g., for $L=512,1024$ or more the period is always $2 L$. This helps to calculate the order parameter. Given an initial distribution of grains, it therefore needs to find out the mean activity over only a period and then average over many initial configurations.

The critical density $\zeta_{c}$ actually has a system size dependence. To study this variation we start with a closed empty lattice of size $L \times L$ and go on adding sand grains one by one at randomly selected lattice sites similar to what is done in an open sandpile. The dynamics of the avalanche is followed for each sand grain added. The mean avalanche size increases with the density of grains in the system and at a certain $\zeta=\zeta_{c}(L)$ depending on the sequence of randomly selected sites at which the grains were dropped, the activity does not stop any more and an "infinite" avalanche continues for ever. In practice, in our simulation we followed an avalanche up to a certain 


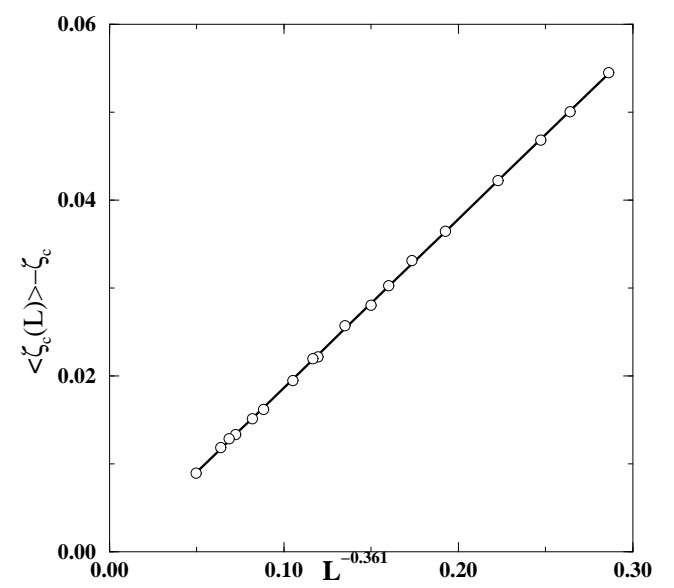

FIG. 3: The variation of the deviation of the critical density $\zeta_{c}(L)$ of a system of size $L$ from its value $\zeta_{c}$ at the infinitely large system is plotted with $L^{-1 / \nu_{\|}}$. For DDFES we obtain $\zeta_{c} \approx 0.4115, \nu_{\|}=1 / 0.361 \approx 2.77$.

relaxation time $T=10^{6}$ for $L<128$ and $T=5 \times 10^{6}$ for $L \geq 128$ to declare the avalanche as infinite. Repeating this simulation a large number of times, every time starting from an empty system, we calculate the average critical density $\left\langle\zeta_{c}(L)\right\rangle$. These values are then extrapolated as: $\left\langle\zeta_{c}(L)\right\rangle=\zeta_{c}+A L^{-1 / \nu_{\|}}$as shown in Fig. 3 to obtain $\zeta_{c}=0.4115 \pm 0.002$ and $1 / \nu_{\|}=0.361$ giving $\nu_{\|} \approx 2.77$. We also esimated $\zeta_{c}$ by the scaling plot of mean avalanche size $\langle s(L)\rangle L^{-0.05}$ vs. $\Delta L^{0.03}$ which is also consistent with our estimate of $\zeta_{c}=0.4115$.

A system of size $L$ is filled initially with density $\zeta$ by randomly distributing $\zeta L^{2}$ grains. We allow the system to evolve up to a relaxation time $T$ after which the activity is measured at every time step. To measure the mean activity $\langle\rho(\Delta, L)\rangle$ for a slightly higher density $\zeta+\delta \zeta$ we take the advantage of the fact that the system dynamics is deterministic. On the same initial distribution of grains corresponding to the density $\zeta$ another $(\delta \zeta) L^{2}$ grains are randomly added. This ensures that if certain density gives sustained activity, its higher density necessarily gives a non-stop activity. These measurements are then repeated for different system sizes. In Fig. 4 we show the scaling of the order parameter on double logarithmic scale. Plotting $\langle\rho(\Delta, L)\rangle L$ with $L^{0.55} \Delta$ we observe a nice data collapse for system sizes $L=128,256$ and 512. Comparing with the Eqn. 1 we conclude $\beta / \nu_{\perp}$ $=1$ and $1 / \nu_{\perp}=0.55$. This implies that $\beta=\nu_{\perp} \approx 1.82$.

The analysis so far enables us to estimate the dynamical exponent $z=\nu_{\|} / \nu_{\perp} \approx 1.52$. This value of the dynamical exponent is directly verified by measuring the survival probability. The survival probability $P(t)$ that the initial activity in a random distribution of grains survives a time $t$ has an exponential distribution as: $P(t) \sim \exp (-t / \tau)$. At the critical point $\zeta_{c}$ the

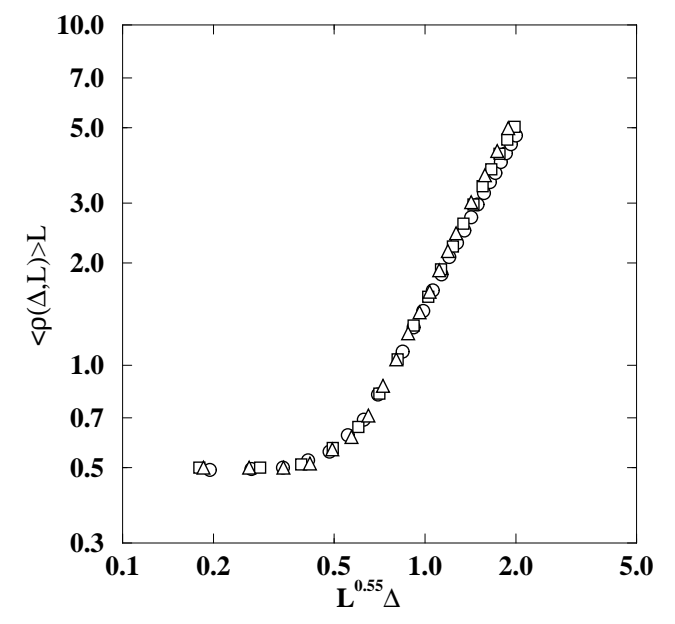

FIG. 4: Scaling of the order parameter $\langle\rho(\Delta, L)\rangle$ with the deviation $\Delta=\zeta-\zeta_{c}$ from the critical point. From this data collapse and Eqn. (1) we find, $\beta / \nu_{\perp}=1$ and $1 / \nu_{\perp}=0.55$.

characteristic time is a function of only the system size as: $\tau(L) \sim L^{z}$ where, $z$ is the dynamical exponent $z=\nu_{\|} / \nu_{\perp}$ of the system. Therefore we calculate the average survival time $\left\langle t_{c}(L)\right\rangle$ which is also proportional to $L^{z}$ at $\zeta_{c}$ for different system sizes. This is done again by dropping grains of sand one by one into a closed system and calculating the life time of the largest avalanche before the system gets locked into an infinite avalanche. Averaging over many initial configurations the largest life-time $\left\langle t_{c}(L)\right\rangle$ is plotted in Fig. 5 for on a double logarithmic scale. The slope of the straight line gives the value for the dynamical exponent $z=1.49 \pm 0.05$ for DDFES compared to 1.52 obtained previously.

In the stochastic directed fixed energy sandpile model the critical density $\zeta_{c}$ is found to be very close to 0.5 . The order parameter has a highly linear variation with $\Delta$ as: $\rho(\Delta)=A \Delta$ where $A \approx 0.46$. A similar to DDFES calculation of the system size dependent critical density after extrapolation $\left\langle\zeta_{c}(L)\right\rangle=\zeta_{c}+A^{\prime} L^{-1 / \nu_{\|}}$gives $\nu_{\|} \approx 1$ and $A^{\prime} \approx 2.4$. The plot of $\langle\rho(\Delta, L)\rangle$ vs. $\Delta$ is a very nice straight line and the plot of data for different system sizes fall on top of one another. We conclude that $\beta \approx 1$ and $\nu_{\perp} \approx \infty$.

The roughneing of the associated interface in our FES models is studied. If $H_{i}(L, t)$ denotes the number of topplings upto the time $t$ then the set of $H_{i}(L, t)$ s for all $i$ represents an interface. For DDFES the width of this interface fluctuates periodically but its average grows as: $W(L, t) \sim L^{\alpha} \mathcal{G}\left(t / L_{i}^{z}\right)$ where we find for $\alpha=0.31$ and $z=1.6$ in comparison to the Linear Interface Model results $\alpha=0.75$ and $z=1.56[22]$.

Finally we study the DFES models on oriented square lattices of rectangular shapes, the longer sides being parallel to the preferred direction. For DDFES model the 


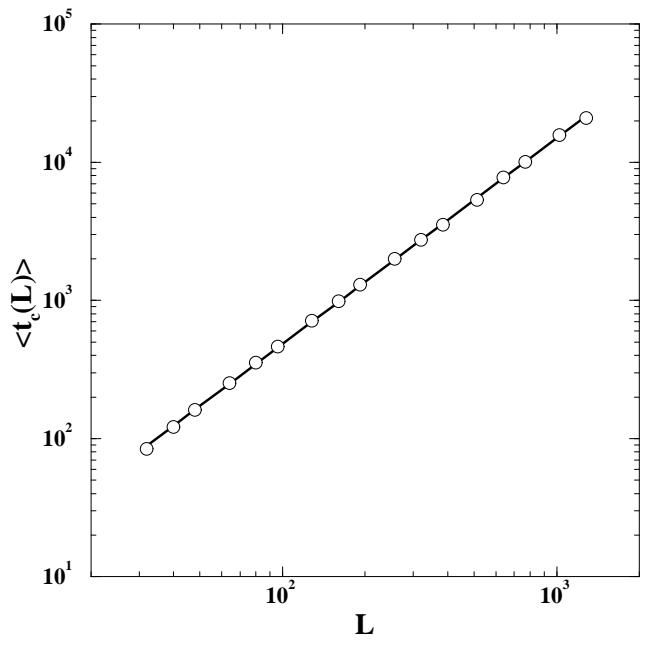

FIG. 5: The average of maximal life-time $\left\langle t_{c}(L)\right\rangle$ of the avalanche prior to the infinite avalanche is plotted with $L$. The slope gives a measure of the dynamical exponent $z=$ 1.49 .

\begin{tabular}{lllll}
\hline \hline Model & $\zeta_{c}$ & $\beta$ & $\nu_{\perp}$ & $\nu_{\|}$ \\
\hline DDFES & 0.4115 & 1.82 & 1.82 & 2.77 \\
SDFES & 0.5 & 1.00 & $\infty$ & 1.00 \\
BTW FES & 2.125 & 0.7 & 0.90 & 1.49 \\
Manna FES & 0.71695 & 0.64 & 0.82 & 1.29 \\
DP & & 0.583 & 0.733 & 1.295 \\
\hline
\end{tabular}

TABLE I: Comparison of critical points and exponents for different models of fixed energy sandpiles. Exponent values for BTW and Manna sandpiles are taken from 12], DP exponents from [21].

TFs are contiguous sites covering the transverse direction in the form of rings. These toppling rings are perfectly stable, once formed they never change in shape. As density increases, the number of such rings increases. On the other hand for SDFES, the toppling sites are randomly scattered throughout the system (Fig. 6).

To summarize, we have studied the directed version of the fixed energy sandpile on the oriented square lattices. Like isotropic FES, our directed FES also shows a continuous phase transition from an absorbed phase to an active phase. Two versions of the model are studied. In the deterministic FES, the grain number configurations are periodic and repeats at regular time interval of $2 L$. For this model the critical points as well as the critical exponents are found to be non-trivial and belong to a new universality class. The other version has the stochastic toppling dynamical rules and exponents of mean-field nature are found for this model.

We thank F. O. Ogundare for some initial discussions.
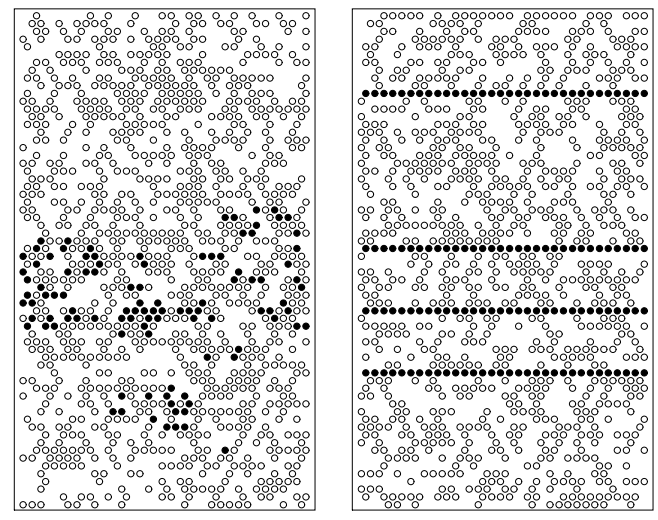

FIG. 6: Two snapshots of height configurations in the directed fixed energy sandpile model on a $32 \times 64$ oriented square lattice, downward direction being the preferred direction. Stochastic DFES is shown on the left where as the deterministic DFES is shown on the right. Active sites are shown by filled circles, open circles denote sites with height 1 and vacant sites are not indicated.

[1] P. Bak, C. Tang and K. Wiesenfeld, Phys. Rev. Lett. 59, 381 (1987); P. Bak, C. Tang and K. Wiesenfeld, Phys. Rev. A 38, 364 (1988).

[2] P. Bak, How Nature Works: The Science of SelfOrganized Criticality, (Copernicus, New York, 1996).

[3] D. Dhar, Studying Self-Organized Criticality with Exactly Solved Models, arXiv:cond-mat/9909009

[4] P. Grassberger and S.S. Manna, J. Phys. (Paris) 51, 1077 (1990).

[5] A. Corral and M. Paczuski, Phys. Rev. Lett., 83, 572 (1999).

[6] D. V. Ktitarev, S. Lübeck, P. Grassberger, and V. B. Priezzhev, Phys. Rev. E 61, 81 (2000).

[7] P. K. Mohanty and D. Dhar, Phys. Rev. Lett. 89, 104303 (2002).

[8] M. De Menech and A. L. Stella, Physica A 309, 289 (2002).

[9] A. Bershadskii and K. R. Sreenivasan, Eur. Phys. J. B 35, 513, 2003.

[10] M. Rossi, R. Pastor-Satorras and A. Vespignani, Phys. Rev. Lett. 85, 1803 (2000).

[11] M. A. Muñoz, R. Dickman, R. Pastor-satorras, A. Vespignani and S. Zapperi, arXiv:cond-mat/0011447

[12] A. Vespignani, R. Dickman, M. A. Muñoz and S. Zapperi, Phys. Rev. E. 62, 4564 (2000).

[13] R. Dickman, M. Alava, M. A. Muñoz, J. Peltola, A. Vespignani and S. Zapperi, Phys. Rev. E. 64, 056104 (2001).

[14] W. Kinzel, in Percolation structures and processes, edited by G. Deutscher, R. Zallan, and J. Adler (Adam Hilger, 1983), vol. 5, pp 425.

[15] D. Dhar and R. Ramaswamy, Phys. Rev. Lett. 63, 1659 (1989).

[16] R. Pastor-Satorras and A. Vespignani, J. Phys. A 33, L33 (2000).

[17] R. Pastor-Satorras and A. Vespignani, Phys. Rev. E. 62, 
6195 (2000).

[18] A. E. Scheidegger, Bull. I.A.S.H. 12 (1), 15 (1967).

[19] B.K. Chakrabarti and S. S. Manna; J. Phys. A. 16, L113 (1983).

[20] F. Bagnoli, F. Cecconi, A. Flammini and A. Vespignani, Europhys. Lett. 63, 512 (2003).
[21] P. Fröjdh, M. Howard and K. B. Lauritsen, Int. J. Phys. C, 15, 1761 (2001).

[22] H. Leschhorn, T. Nattermann, S. Stepanow and L.-H. Tang, Ann, Phys. (N.Y.) 6, 1 (1997). 